\title{
Low-loss and single-mode tapered hollow-core waveguides optically coupled with interband and quantum cascade lasers
}

\author{
Marilena Giglio, ${ }^{a, b}$ Pietro Patimisco, ${ }^{a, b}$ Angelo Sampaolo, ${ }^{a, b}$ Jason M. Kriesel, ${ }^{c}$ Frank K. Tittel, ${ }^{b}$ and Vincenzo Spagnolo ${ }^{a, *}$ \\ aUniversity and Politecnico of Bari, PolySense Lab-Dipartimento Interateneo di Fisica, Bari, Italy \\ ${ }^{\mathrm{b}}$ Rice University, Department of Electrical and Computer Engineering, Houston, Texas, United States \\ 'Opto-Knowledge Systems, Inc. (OKSI), Torrance, California, United States
}

\begin{abstract}
We report single-mode midinfrared laser beam delivery through a 50-cm-long tapered hollow-core waveguide (HCW) having bore diameter linearly increasing from 200 to $260 \mu \mathrm{m}$. We performed theoretical calculations to identify the best HCW-laser coupling conditions in terms of optical losses and single-mode fiber output. To validate our modeling, we coupled the HCW with an interband cascade laser and four quantum cascade lasers with their emission wavelengths spanning 3.5 to $7.8 \mu \mathrm{m}$, using focusing lenses with different focal lengths. With the best coupling conditions, we achieved single-mode output in the investigated 3.5 to $7.8 \mu \mathrm{m}$ spectral range, with minimum transmission losses of $1.27 \mathrm{~dB}$ at $6.2 \mu \mathrm{m}$. $\odot 2017$ Society of Photo-Optical Instrumentation Engineers (SPIE) [DOI: 10.1117/1.OE.57.1.011004]
\end{abstract}

Keywords: fiber; quantum cascade laser; interband cascade laser; single-mode delivery; optical coupling.

Paper 170818SS received May 30, 2017; accepted for publication Aug. 1, 2017; published online Aug. $24,2017$.

\section{Introduction}

Hollow-core waveguides (HCWs) are optical fibers that guide light within a cylindrical hollow region contained in a tube with walls composed of metallic/dielectric highreflective layers. Unlike the conventional light guiding scheme where a high-refractive-index core is surrounded by a low-refractive-index cladding, in HCWs the light is guided through a low-index core (air) surrounded by high-index cladding layers. HCWs have become increasingly important in applications, such as trace gas sensing and the opto-fluidic. ${ }^{1-4} \mathrm{HCWs}$ recently found applications as a laser beam delivery device to guide light in the midinfrared spectral range due to the lack of low-absorption materials for solid-core fibers. ${ }^{5-7}$ Typically, these types of HCWs consist of a fused silica capillary tube of fixed bore size with a reflective silver film deposited on the inner surface. A thin film of silver iodine is deposited on the silver layer. The dielectric film thickness determines the optical response of the $\mathrm{HCW}$, leading to the design of an optimized structure for a desired spectral range of operation. ${ }^{7}$ As for all waveguides, the propagation losses and the mode profile play a critical role in their operation. ${ }^{8}$ A single-mode beam profile output can be obtained when the bore diameter is up to 50 times larger than the light wavelength. ${ }^{9,10}$ Silica-based HCWs with a bore diameter of $300 \mu \mathrm{m}$ have recently been reported that can deliver optical guidance in the 8 to $11 \mu \mathrm{m}$ spectral range with a single spatial mode at the output. ${ }^{10-12}$ In the 3.7 to $7.6 \mu \mathrm{m}$ spectral range, low-loss and single-mode laser beam delivery through silica-based HCWs with a bore diameter of $200 \mu \mathrm{m}$ was demonstrated. ${ }^{13} \mathrm{HCWs}$ can also act as an efficient, mid-IR spatial modal beam filter with single-

*Address all correspondence to: Vincenzo Spagnolo, E-mail: vincenzoluigi. spagnolo@poliba.it mode Gaussian-like beam output. ${ }^{14,15}$ These results led to the implementation of HCWs in gas spectroscopic sensor systems to improve the spatial quality of laser beams, which can affect the ultimate performance of gas sensors. For example, HCWs with a bore diameter of $300 \mu \mathrm{m}$ have been implemented in quartz-enhanced photoacoustic sensors employing quantum cascade lasers (QCLs). The beam filtering provided by the fiber enables high-performance detection of $\mathrm{SF}_{6}$ gas traces with concentrations down to a few tens of part-per-trillion in volume. ${ }^{10-12,16}$

Tapered HCWs, i.e., with the diameter linearly varying along the HCW length, have been proposed as a clever way to provide single-mode operation and facilitate the optical coupling alignment using the larger bore size as input. ${ }^{17,18}$ The propagation losses and the quality of the $\mathrm{HCW}$ output beam are strongly influenced by the laser-HCW optical coupling conditions. The selection of the focal length of the coupling lens is mainly determined by the laser beam diameter as well as the wavelength and the HCW bore diameter. ${ }^{17}$ However, a single focusing lens cannot guarantee optimal coupling conditions for a wide wavelength range.

In this work, we investigate the properties of an $\mathrm{HCW}$ with a tapered bore profile, i.e., with the bore diameter linearly increasing from $2 a_{1}=200 \mu \mathrm{m}$ to $2 a_{2}=260 \mu \mathrm{m}$ for an $L=50 \mathrm{~cm}$ fiber length. The internal high-reflective surface is composed of a silver/silver iodine double layer. The tapered fiber was coupled with an interband cascade laser (ICL) emitting at $3.5 \mu \mathrm{m}$ and four QCLs emitting at 4.6, 5.2, 6.2, and $7.8 \mu \mathrm{m}$, to investigate the performance of the $\mathrm{HCW}$ in a wide spectral range. The launch conditions, the propagation losses, and the beam quality at the waveguide output were analyzed and evaluated using both fiber sides 
as HCW input (i.e., coupling into the smaller end versus coupling into the larger end).

\section{Theoretical Model}

When optical coupling between a collimated laser beam and the HCW is obtained using a focusing lens, the propagation losses and the beam quality at the waveguide exit are affected by the input laser beam quality, the size as well as the focal length of the coupling lens. The propagation of the guided light within an HCW can be treated by the ray-optics model, assuming that an optical beam can be guided by zig-zag reflections at the guiding walls. The model is based on tracking consecutive reflections of a bundle of optical rays within a cone at the waveguide entrance and propagating through the waveguide. ${ }^{18}$ Such a model allows the determination of both the number of reflections at the waveguide wall and the angle of incidence for each reflection. However, to determine how the coupling conditions are correlated to waveguide losses and to the output-beam quality, we exploited the theoretical model proposed in Ref. 9. This model is based on Maxwell's equations and Gaussian optics assumptions. Considering a diffraction-limited collimated Gaussian beam with a radius $w$ illuminating a lens with a focal length $f$ and radius of curvature $R$, the waist radius $w_{0}$ at the focal plane is given by

$$
w_{0}=\frac{w}{\sqrt{1+\left(\frac{\pi w^{2}}{\lambda R}\right)^{2}}},
$$

and the focal length $f$ at different laser wavelengths $\lambda$ is given by

$$
f=\frac{R}{1+\left(\frac{\lambda R}{\pi w^{2}}\right)^{2}} .
$$

Hence, by measuring the size of the collimated beam, it is possible to estimate the waist radius at the waveguide entrance. When a Gaussian beam is focused into a hollow waveguide, the best coupling condition is obtained by maximizing the input laser mode coupling into the $\mathrm{HE}_{11}$ waveguide hybrid mode, providing the lowest theoretical losses and characterized by a Gaussian-like optical power distribution. ${ }^{19-21}$ Spatial profiles of $\mathrm{HE}_{1 \mathrm{~m}}$ waveguide modes can be approximated by the zero-order Bessel functions $J\left(u_{1 \mathrm{~m}} r / a\right)$, where $a$ is the bore radius and $u_{1 \mathrm{~m}}$ is the $m$ 'th root of the zero-order Bessel function. If a Gaussian beam is focused to a waist $w_{0}$ at the fiber input plane, the intensity distribution is given by

$G(r)=G_{0} e^{-\frac{r^{2}}{w_{0}^{2}}}$

and the amount of the optical power coupled with the $m$ 'th waveguide mode can be expressed in terms of the power coupling efficiency defined as

$\eta_{1 m}=\frac{\left|\int_{0}^{a} G(r) J\left(u_{1 \mathrm{~m}} \frac{r}{a}\right) r \mathrm{~d} r\right|^{2}}{\int_{0}^{a} G^{2}(r) r \mathrm{~d} r \int_{0}^{a} J^{2}\left(u_{1 \mathrm{~m}} \frac{r}{a}\right) r \mathrm{~d} r}$.

Hence, the $\eta_{1 \mathrm{~m}}$ coefficients depend on $w_{0} / a$. The highest coupling efficiency to the $\mathrm{HE}_{11}$ mode is obtained for $w_{0} / a=0.64 .{ }^{17}$ Values significantly lower than 0.64 guarantee low propagation losses, but cannot ensure single-mode output conditions, since a significant fraction of the input power is coupled to higher order modes that can propagate to the waveguide exit, whereas operating at $w_{0} / a>0.64$ leads to higher losses but the HCW output beam remains single mode. ${ }^{17}$ For a collimated beam radius $w=1 \mathrm{~mm}$ and coupling lenses with $f=25,50$, and $75 \mathrm{~mm}$, using Eqs. (1)-(4), we calculated the $\eta_{11}$ coupling efficiency as a function of the laser wavelength, considering $a_{1}$ or $a_{2}$ as input bore radius. The results are shown in Fig. 1.

Using $f=50 \mathrm{~mm}$, it is possible to keep the theoretical coupling efficiency $\eta_{11}>90 \%$ in the 3.9 to $7.8 \mu \mathrm{m}$ spectral range, by appropriate selection of the smaller $\left(a_{1}\right)$ or the larger $\left(a_{2}\right)$ bore side as the HCW input [see Fig. 1(b)], whereas for $7.8 \mu \mathrm{m}<\lambda<8.5 \mu \mathrm{m}$, values of $\eta_{11}>90 \%$ are obtained using $f=25 \mathrm{~mm}$ and $a_{1}$ as the input side [see Fig. 1(a)]. Instead, for $3 \mu \mathrm{m}<\lambda<3.9 \mu \mathrm{m}$ it is feasible keeping $\eta_{11}>90 \%$, with $f=75 \mathrm{~mm}$ and $a_{1}$ as input side [see Fig. 1(c)].

To estimate the propagation losses, we employed the equation derived by Miyagi and Kawakami in Ref. 22 to determine the attenuation coefficients $\alpha_{1 \mathrm{~m}}$ for the $\mathrm{HE}_{1 \mathrm{~m}}$ waveguide modes, considering that the inner core radius $a(z)$ varies linearly along the waveguide as

$a(z)=\frac{a_{\text {output }}-a_{\text {input }}}{L} z+a_{\text {input }}$,

where $a_{\text {input }}$ and $a_{\text {output }}$ are the bore radii at the HCW input and output, respectively.
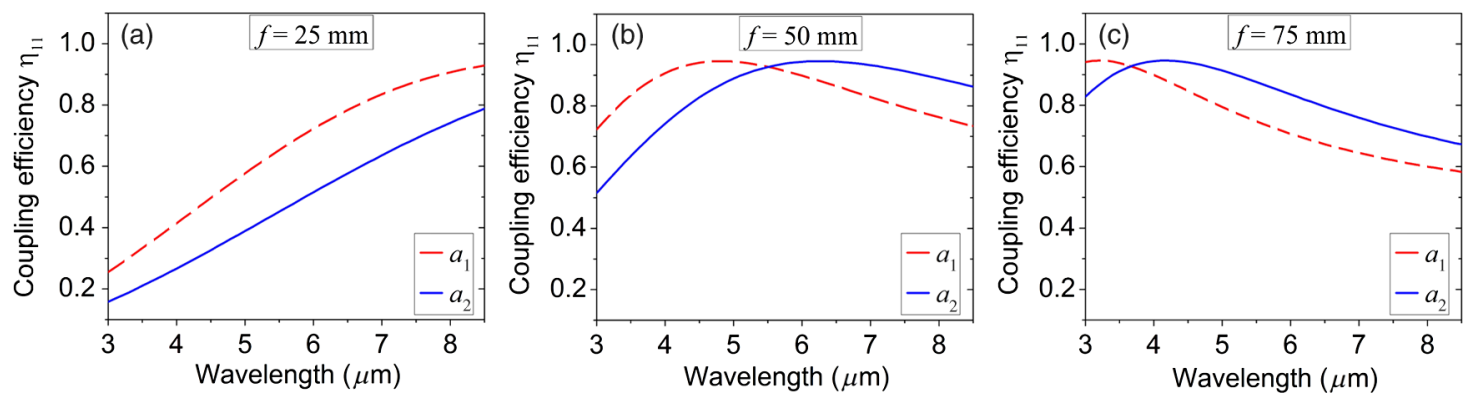

Fig. 1 (a-c) $\eta_{11}$ coupling efficiency calculated as a function of the laser wavelength, when the input beam is coupled through the $a_{1}$ (red dashed line) or $a_{2}$ (blue solid line) input side, using a coupling lens with $f=25,50$, or $75 \mathrm{~mm}$, respectively. 
The theoretical propagation losses can be calculated by using the following expression: ${ }^{9}$

$L_{p}(\mathrm{~dB})=-10 \log _{10}\left\{\sum_{m} \eta_{1 \mathrm{~m}} \exp \left[-2 \int_{0}^{L} \alpha_{1 \mathrm{~m}}(z) \mathrm{d} z\right]\right\}$,

where the attenuation coefficients are given by

$$
\begin{aligned}
\alpha_{1 \mathrm{~m}} & =\left(\frac{u_{1 \mathrm{~m}}}{2 \pi}\right)^{2} \frac{\lambda^{2}}{a(z)^{3}} \frac{n}{n^{2}+k^{2}} \frac{1}{2}\left(1+\frac{n_{d}^{2}}{\sqrt{n_{d}^{2}-1}}\right)^{2} \\
& =K \frac{u_{1 \mathrm{~m}}^{2} \lambda^{2}}{a(z)^{3}}
\end{aligned}
$$

with

$$
K=\left(\frac{1}{2 \pi}\right)^{2} \frac{n}{n^{2}+k^{2}} \frac{1}{2}\left(1+\frac{n_{d}^{2}}{\sqrt{n_{d}^{2}-1}}\right)^{2} .
$$

Here, $n$ and $k$ are the real and imaginary parts of the complex index of the metallic layer, while $n_{d}$ is the refractive index of the dielectric film. By combining Eqs. (5)-(8), $L_{p}$ can be written as

$$
\begin{aligned}
& L_{p}(\mathrm{~dB}) \\
& \quad=-10 \log _{10}\left[\sum_{m} \eta_{1 \mathrm{~m}} \exp \left(-2 K u_{1 \mathrm{~m}}^{2} \lambda^{2} L \frac{a_{\text {input }}+a_{\text {output }}}{2 a_{\text {input }} a_{\text {output }}}\right)\right] .
\end{aligned}
$$

While the attenuation coefficients do not change whether $a_{1}$ or $a_{2}$ is selected as the HCW input core size, the propagation losses $L_{p}$ are affected by which HCW side is chosen, since the coupling efficiency depends on the $w_{0} / a_{i}$ ratio. These dependences cannot be extracted by the standard ray-optics model, for which propagation losses are not dependent on input tapered HCW bore radius. ${ }^{18}$ Using Eq. (9), we calculated $L_{p}$ values as a function of the wavelength with $f=25 \mathrm{~mm}, f=50 \mathrm{~mm}$, and $f=75 \mathrm{~mm}$, respectively, as reported in Figs. 2(a)-2(c). In Figs. 2(d)-2(f), we reported the corresponding $w_{0} / a_{i}$ ratios. For the wavelength dependence of $n, k$, and $n_{d}$ we used relations as reported in Ref. 23.

The simulation results reported in Fig. 2(b) show that using a lens with $f=50 \mathrm{~mm}$, the appropriate selection of the HCW input side provides propagation losses $<1 \mathrm{~dB}$ in the 3 to $7 \mu \mathrm{m}$ spectral range. However, minimizing the optical losses does not imply the achievement of a single-mode HCW output condition in the entire investigated spectral range. Indeed, as shown in Fig. 2(e), $w_{0} / a$ values close to the optimum 0.64 are achieved in the 4 to $7 \mu \mathrm{m}$ spectral range, while for $\lambda<4 \mu \mathrm{m}$, even if using the lowest core size $\left(a_{1}\right)$ as $\mathrm{HCW}$ input side, $w_{0} / a_{1}$ values substantially lower than 0.64 are extracted, and therefore do not guarantee the achievement of single-mode output. A lens with a longer focal length provides higher $w_{0} / a$ values and more feasible single-mode output conditions for $\lambda<4 \mu \mathrm{m}$. In Figs. 2(c) and 2(f), we reported the calculated optical losses and the $w_{0} / a_{i}$ ratios when using a coupling lens with $f=75 \mathrm{~mm}$. For wavelengths $<5.5 \mu \mathrm{m}$, losses as low as $1 \mathrm{~dB}$ and $w_{0} / a_{i}$ values approximating to 0.64 could be achieved. On the other hand, for $\lambda>7 \mu \mathrm{m}, f=25 \mathrm{~mm}$ and the selection of $a_{1}$ as the HCW input side guarantees that $L p<1 \mathrm{~dB}$ and $w_{0} / a_{1}$ approaches 0.64 , as shown in Figs. 2(a) and 2(d).

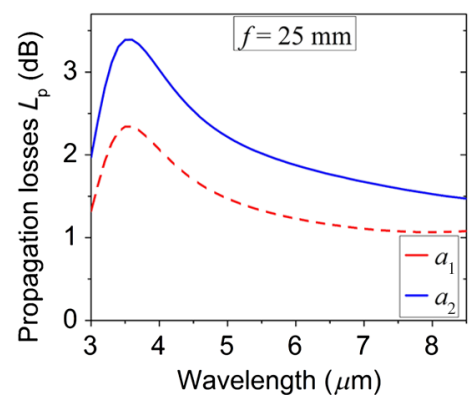

(a)

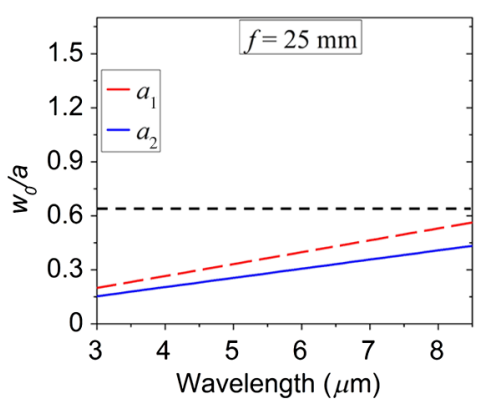

(d)

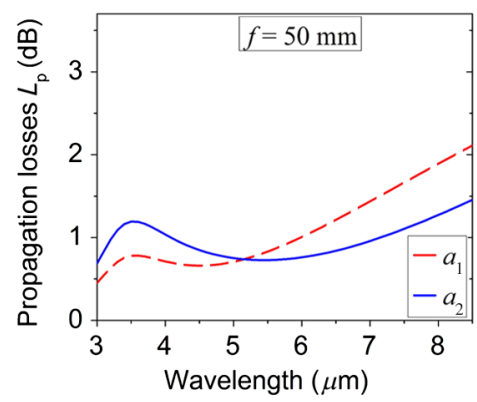

(b)

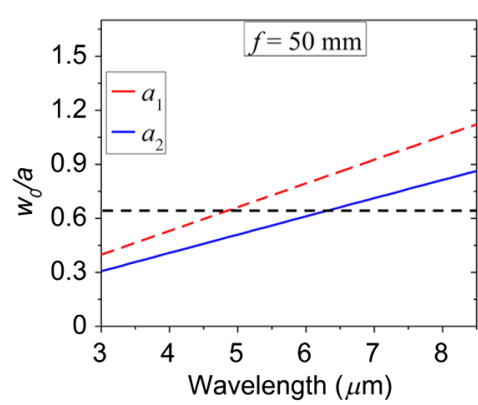

(e)

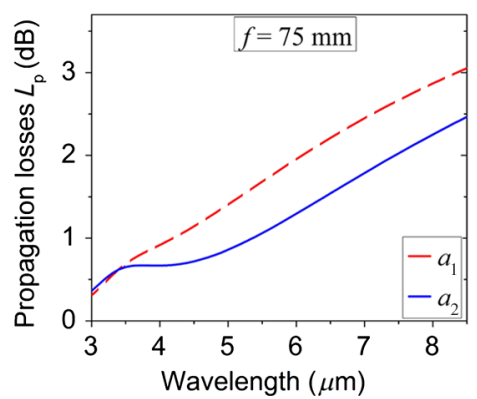

(c)

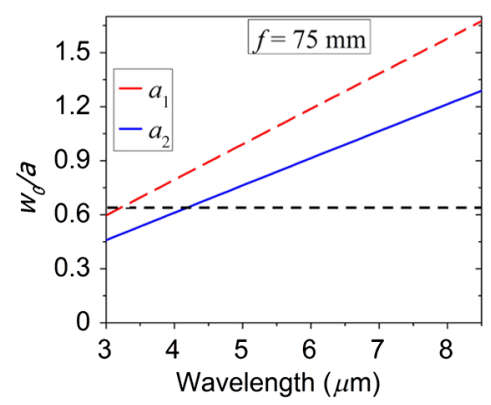

(f)

Fig. 2 (a-c) Propagation losses $L_{p}$ as a function of the wavelength when the laser beam is focused focused into $a_{1}$ (red dashed line) or $a_{2}$ (blue solid line), using a lens with $f=25,50$, or $75 \mathrm{~mm}$, respectively. (d-f) The $w_{0} / a_{i}$ ratios calculated as a function of the wavelength when the laser beam is focused into $a_{1}$ (red dashed line) or $a_{2}$ (blue solid line), using a lens with $f=25,50$, or $75 \mathrm{~mm}$. The dashed horizontal lines mark the $w_{0} / a_{i}$ optimum value of 0.64 . 


\section{Beam Profiles at the Hollow-Core Waveguide Waveguide Exit}

The tapered HCW employed in this work consists of a fused silica capillary tube having a bore diameter linearly increasing from 200 to $260 \mu \mathrm{m}$ for a length of $50 \mathrm{~cm}$. A sketch of the section of the fiber along its axis is shown in Fig. 3.

The fabrication technique used to realize such a fiber and deposit high-quality silver (Ag) and silver iodide (AgI) films on the capillary bore is known as a dynamic liquid deposition phase. ${ }^{7}$ Custom tapered glass tubing was produced at a commercial glass drawing facility, based on OKSI Inc. proprietary design and specifications. The fused silica capillary inner surface is sensitized to improve adhesion of the silver film to be deposited and reduce the time needed for this procedure. A metallic coating is successively deposited by flowing a silver solution through the capillary. Finally, an iodine solution is pumped through the coated waveguide, which

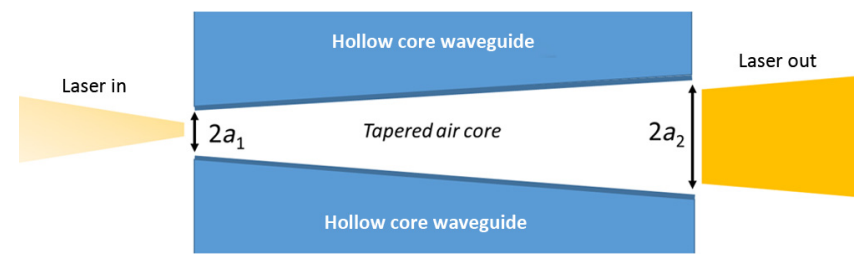

Fig. 3 Sketch of the longitudinal section of the tapered HCW. The air core diameter increases linearly from $2 a_{1}=200 \mu \mathrm{m}$ to $2 a_{1}=260 \mu \mathrm{m}$, for a total fiber length of $50 \mathrm{~cm}$.

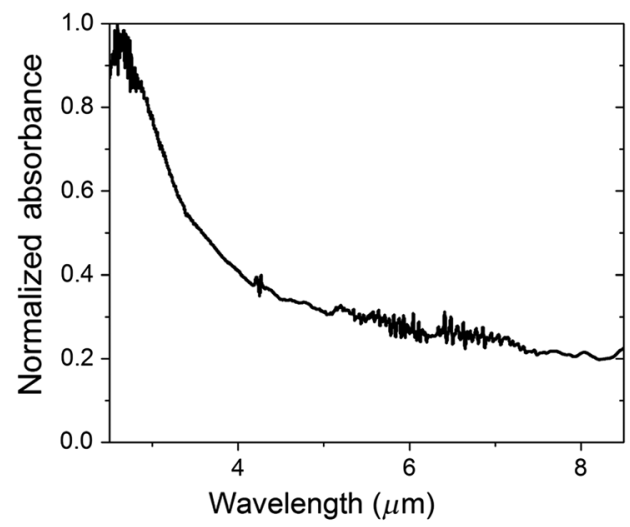

Fig. 4 Absorbance profile of a 7-cm long tapered HCW segment. The diameter of this tapered HCW segment ranges from 190 to $200 \mu \mathrm{m}$ over its length of $7 \mathrm{~cm}$. The thickness of the dielectric layer is optimized for wavelengths $>5 \mu \mathrm{m}$. reacts with the metallic substrate, resulting in the conversion of the Ag into AgI. All the fabrication steps are performed by using a peristaltic pump and a flow rate selected to guarantee a high coating uniformity. The dielectric layer thickness of the tapered HCW was designed with the aim of optimizing the fiber performance in the 4 to $10 \mu \mathrm{m}$ spectral range. The $50-\mathrm{cm}$ long fiber used for the studies reported here was cut from a coated fiber that was originally $2.5-\mathrm{m}$ long. Figure 4 shows the FTIR absorption spectrum measured in the 2.5 to $8.5 \mu \mathrm{m}$ spectral range for a $7-\mathrm{cm}$ long tapered fiber segment that was cut from the same longer stock fiber near the small side of the HCW and is thus a good representation of the spectral absorption of the fiber used in this work.

The measured absorbance changes slightly from 8 to $5 \mu \mathrm{m}$, while it increases rapidly at shorter wavelengths. Therefore, higher losses are expected to occur at wavelengths $<5 \mu \mathrm{m}$.

The performance of the tapered 50-cm-long HCW was experimentally investigated by employing three mid-IR distributed feedback quantum cascade lasers (DFB-QCLs), one mid-IR external cavity quantum cascade laser (ECQCL), and one ICL, emitting at wavelengths of $7.8 \mu \mathrm{m}$ (Corning, DFB-QCL), $6.2 \mu \mathrm{m}$ (Daylight, EC-QCL), $5.2 \mu \mathrm{m}$ (Hamamatsu, DFB-QCL), $4.6 \mu \mathrm{m}$ (AdTech, DFBQCL), and $3.5 \mu \mathrm{m}$ (Nanoplus, ICL). In Figs. 5(a)-5(e), the beam profiles acquired at a distance of $7 \mathrm{~cm}$ from the output of these lasers using a $124 \times 124$ pixels infrared pyro-camera (Ophir, Spiricon), providing a spatial resolution of $100 \mu \mathrm{m}$, are shown.

The five lasers exhibit low spatial beam qualities with asymmetric far-field optical power distributions. In particular, the beam profiles of the QCLs emitting at 7.8 and $4.6 \mu \mathrm{m}$ and of the ICL at $3.5 \mu \mathrm{m}$ show the presence of multiple lobes. For this reason, we calculated the second-order moment of the intensity distribution along both $x$ and $y$ directions, as reported in Ref. 14 to extract the beam diameters. For the five lasers, an average value for the beam radius of $w=1 \mathrm{~mm}$ was estimated. The employed laser sources were optically coupled with the tapered HCW using the input $a_{1}$ or $a_{2}$. The HCW was kept straight in all the measurements. The experimental setup we used is schematically represented in Fig. 6 and is similar to the one reported in Ref. 14.

A ZnSe coupling lens with an AR coating in the 3 to $12 \mu \mathrm{m}$ spectral range was used to focus the collimated laser beams into the tapered HCW. We selected lenses with $f=25,50$, and $75 \mathrm{~mm}$. For each focal length, we used the theoretical model described in Sec. 2 to evaluate the coupling efficiency and the $w_{0} / a_{i}$ ratios when the

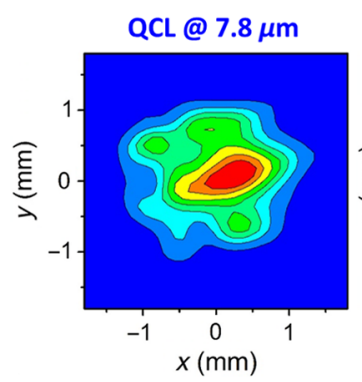

(a)

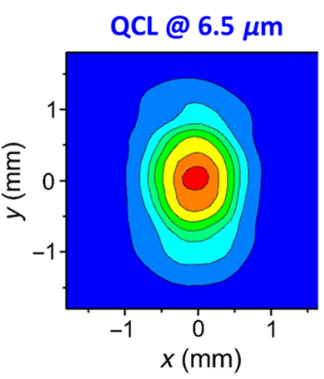

(b)

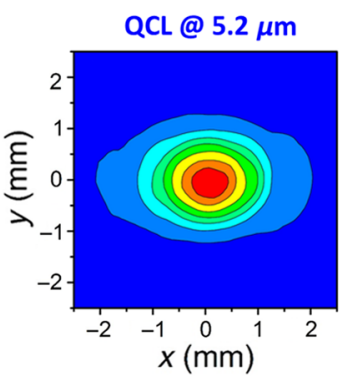

(c)

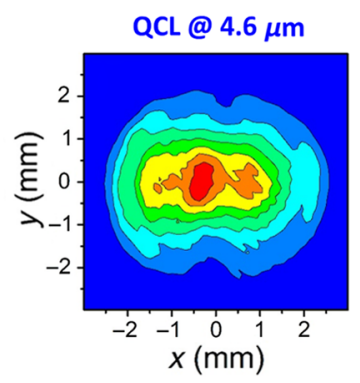

(d)

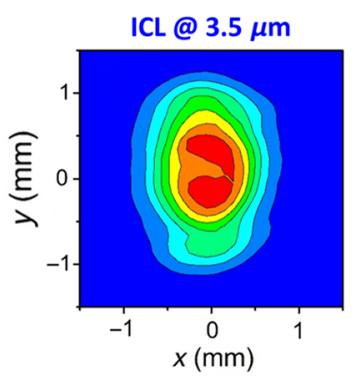

(e)

Fig. 5 Beam profiles acquired by positioning the pyrocamera $7 \mathrm{~cm}$ from the output of the (a) $7.8 \mu \mathrm{m}$ QCL,

(b) $6.2 \mu \mathrm{m} \mathrm{QCL}$, (c) $5.2 \mu \mathrm{m} \mathrm{QCL}$, (d) $4.6 \mu \mathrm{m} \mathrm{QCL}$, and (e) $3.5 \mu \mathrm{m}$ ICL. 


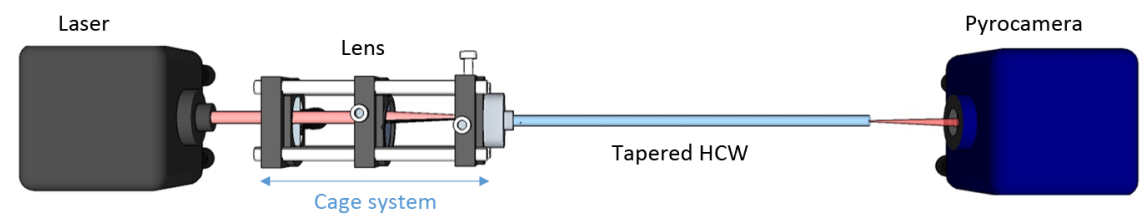

Fig. 6 Schematic of the experimental setup used to optically couple the laser sources to the tapered HCW by employing a ZnSe focusing lens with AR coating in the 3 to $12 \mu \mathrm{m}$ spectral range.

laser beams are coupled to the $a_{1}$ or $a_{2} \mathrm{HCW}$ sides. In Table 1, for each wavelength, we reported the focal length providing the best coupling conditions, i.e., highest coupling efficiencies $\eta_{11}$ and $w_{0} / a_{i}$ ratios as close as possible to the optimum value of 0.64 .

Table 1 Focal lengths $f$ providing the best coupling conditions for each laser source having average beam diameter $2 w$ and corresponding $\eta_{11}$ and $w_{0} / a_{i}$ values, using $a_{1}$ or $a_{2}$ as fiber input side.

\begin{tabular}{|c|c|c|c|c|c|c|}
\hline & & $\begin{array}{c}7.8 \mu \mathrm{m} \\
\mathrm{QCL}\end{array}$ & $\begin{array}{l}6.2 \mu \mathrm{m} \\
\mathrm{QCL}\end{array}$ & $\begin{array}{c}5.2 \mu \mathrm{m} \\
\mathrm{QCL}\end{array}$ & $\begin{array}{c}4.6 \mu \mathrm{m} \\
\mathrm{QCL}\end{array}$ & $\begin{array}{c}3.5 \mu \mathrm{m} \\
\mathrm{ICL}\end{array}$ \\
\hline & $2 w$ & $1.9 \mathrm{~mm}$ & $2.3 \mathrm{~mm}$ & $2.8 \mathrm{~mm}$ & $3.5 \mathrm{~mm}$ & $1.9 \mathrm{~mm}$ \\
\hline$a_{1}$ & $f$ & $25 \mathrm{~mm}$ & $50 \mathrm{~mm}$ & $50 \mathrm{~mm}$ & $75 \mathrm{~mm}$ & $75 \mathrm{~mm}$ \\
\hline & $\eta_{11}$ & $89.6 \%$ & $88.6 \%$ & $94.0 \%$ & $83.6 \%$ & $93.8 \%$ \\
\hline & $w_{0} / a_{1}$ & 0.52 & 0.82 & 0.69 & 0.91 & 0.70 \\
\hline$a_{2}$ & $f$ & $25 \mathrm{~mm}$ & $50 \mathrm{~mm}$ & $75 \mathrm{~mm}$ & $75 \mathrm{~mm}$ & $75 \mathrm{~mm}$ \\
\hline & $\eta_{11}$ & $72.2 \%$ & $94.6 \%$ & $89.9 \%$ & $93.6 \%$ & $91.1 \%$ \\
\hline & $w_{0} / a_{2}$ & 0.40 & 0.63 & 0.80 & 0.70 & 0.54 \\
\hline
\end{tabular}

Theoretically, it is possible to reach coupling efficiencies with the $\mathrm{HE}_{11}$ waveguide mode $>89 \%$, by selecting the right input side.

The laser beam profiles, acquired with the pyrocamera located $4 \mathrm{~cm}$ from the tapered HCW output, at the coupling conditions reported in Table 1, are shown in Figs. 7(a)-7(e) and 7(f)-7(j), respectively, when $a_{1}$ or $a_{2}$ is used as the fiber input side.

Despite the low spatial quality and, in some cases, multimodal intensity distribution of the input laser beams, the tapered HCW provides single-mode circular-symmetric Gaussian-like outputs in the 3.5 to $7.8 \mu \mathrm{m}$ investigated spectral range, whenever using $a_{1}$ or $a_{2}$ as the fiber input side. Thereby, the selection of the input side giving the best coupling conditions requires the investigation of the related optical losses. The spatial quality of the laser beam coming out of the waveguide exit can be quantified by calculating $M$-squared parameter. By collecting HCW output-beam profiles at different distances from the HCW end, we calculated the widths of the beam, by using the second-moment-based width method, starting from $2 \mathrm{~cm}$ away from the HCW end. In this way, it is possible to estimate the half-angle beam divergence of the beam coming out of the waveguide exit. ${ }^{6}$ This value can be compared with the theoretical half-angle beam divergence $\theta_{\text {theo }}=2.405 \lambda / 2 \pi a,{ }^{7}$ calculated by assuming that only the lowest-order HCW mode

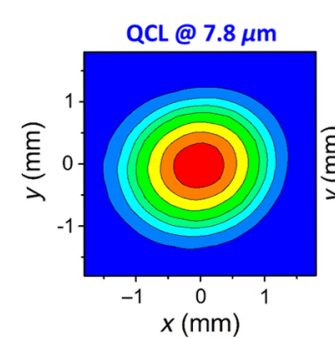

(a)

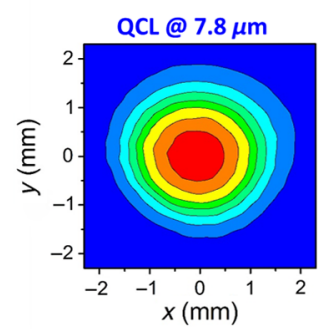

(f)

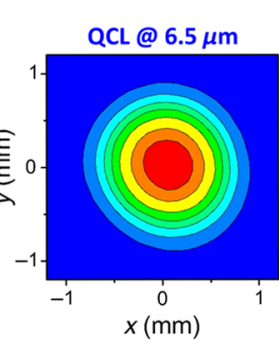

(b)

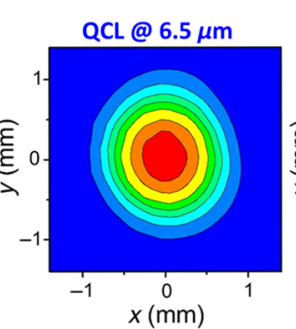

(g)

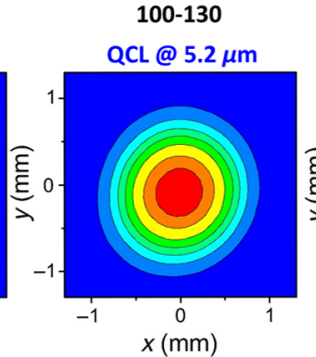

(c)

$130-100$

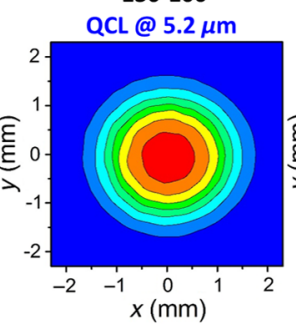

(h)

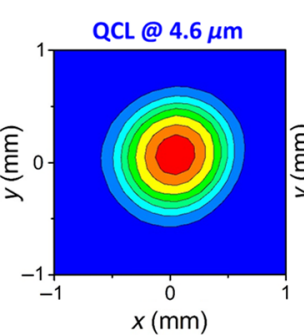

(d)

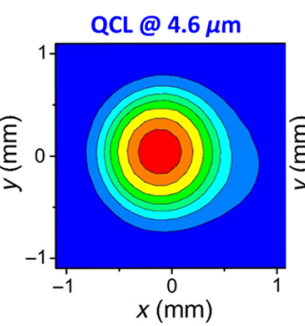

(i)

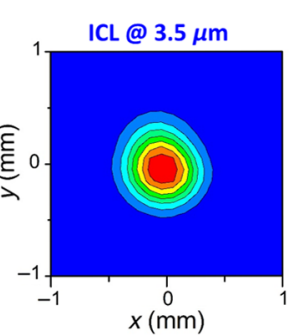

(e)

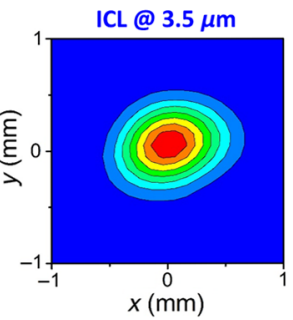

(j)

Fig. 7 (a-e) Beam profiles of the five lasers acquired $4 \mathrm{~cm}$ from the tapered HCW exit when $\mathrm{a}_{1}$ is used as input side. (f-j) Beam profiles of the five lasers acquired $4 \mathrm{~cm}$ from the tapered HCW exit when $a_{2}$ is used as the input side. The coupling conditions are reported in Table 1. 
propagates, and the $\mathrm{M}$-squared can be expressed as the ratio $M^{2}=\theta / \theta_{\text {theo. }}$. In Table 2 , we reported both the half-angle beam divergence and the calculated $\mathrm{M}$-squared values.

Apart from the $3.5 \mu \mathrm{m}$-ICL coupled by using $a_{2}$ as the fiber input side, M-squared values in the 1.06 to 1.16 range were obtained, confirming the achievement of a single-mode output close to a pure Gaussian beam at the waveguide exit.

\section{Optical Losses}

We calculated the total losses $L_{t}$ for each laser source, by measuring the optical power at the input and at the output of the tapered HCW at the coupling conditions reported in Table 1. The histogram in Fig. 8 shows the comparison between the total losses measured are compared when $a_{1}$ (red and white bars) or $a_{2}$ (blue bars) is selected as the input side.

In agreement with the FTIR absorbance measurements reported in Fig. 3, the total losses increase at $3.5 \mu \mathrm{m}$. It is worth noting that, for each laser source we reached lower losses when the tapered HCW is coupled using the input side giving the larger coupling efficiency $\eta_{11}$ in Table 1.

In Table 3, the best single-mode coupling conditions are summarized for each laser source, i.e., coupling lens focal length $f$, input side $a_{i}$, coupling efficiency $\eta_{11}, w_{0} / a_{i}$ ratio, and theoretical propagation losses $L_{p}$, as well as corresponding measured total losses $L_{t}$.

In the 4.6 to $7.8 \mu \mathrm{m}$ spectral range, we obtained discrepancies between the losses $L_{t}-L_{p}<1.1 \mathrm{~dB}$. At $3.5 \mu \mathrm{m}$, this discrepancy increases up to $1.75 \mathrm{~dB}$. These results suggest

Table 2 Half-angle beam divergences and related $M$-squared values, for each laser coupled by using $a_{1}$ or $a_{2}$ as fiber input side.

\begin{tabular}{|c|c|c|c|c|c|c|}
\hline & & $\begin{array}{c}7.8 \mu \mathrm{m} \\
\mathrm{QCL}\end{array}$ & $\begin{array}{c}6.2 \mu \mathrm{m} \\
\mathrm{QCL}\end{array}$ & $\begin{array}{c}5.2 \mu \mathrm{m} \\
\mathrm{QCL}\end{array}$ & $\begin{array}{c}4.6 \mu \mathrm{m} \\
\mathrm{QCL}\end{array}$ & $\begin{array}{c}3.5 \mu \mathrm{m} \\
\text { ICL }\end{array}$ \\
\hline \multirow[t]{2}{*}{$a_{1}$} & $\theta$ & $34.3 \mathrm{mrad}$ & $27.7 \mathrm{mrad}$ & $22.3 \mathrm{mrad}$ & $18.7 \mathrm{mrad}$ & $15.3 \mathrm{mrad}$ \\
\hline & $M^{2}$ & 1.15 & 1.16 & 1.12 & 1.06 & 1.14 \\
\hline \multirow[t]{2}{*}{$a_{2}$} & $\theta$ & $26.6 \mathrm{mrad}$ & $20.2 \mathrm{mrad}$ & $16.4 \mathrm{mrad}$ & $14.5 \mathrm{mrad}$ & $18.7 \mathrm{mrad}$ \\
\hline & $M^{2}$ & 1.16 & 1.10 & 1.07 & 1.07 & 1.38 \\
\hline
\end{tabular}

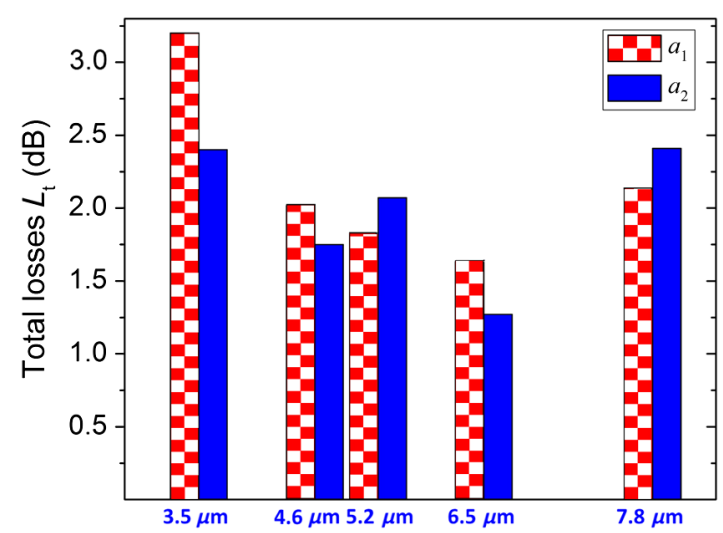

Fig. 8 Total losses $L_{t}$ measured for each laser source, when $a_{1}$ (red checked bars) or $a_{2}$ (blue bars) was used as the HCW input side.
Table 3 Coupling lens focal length $f$, average beam diameter $2 w$, input HCW side radius $a_{i}$, theoretical coupling efficiency $\eta_{11}$, theoretical $w_{0} / a_{i}$ ratio, theoretical propagation losses $L_{p}$ and measured total losses $L_{t}$, at the corresponding best coupling conditions for each investigated laser source.

\begin{tabular}{lccccc}
\hline & $\begin{array}{c}7.8 \mu \mathrm{m} \\
\mathrm{QCL}\end{array}$ & $\begin{array}{c}6.2 \mu \mathrm{m} \\
\text { QCL }\end{array}$ & $\begin{array}{c}5.2 \mu \mathrm{m} \\
\text { QCL }\end{array}$ & $\begin{array}{c}4.6 \mu \mathrm{m} \\
\text { QCL }\end{array}$ & $\begin{array}{c}3.5 \mu \mathrm{m} \\
\mathrm{ICL}\end{array}$ \\
\hline$f$ & $25 \mathrm{~mm}$ & $50 \mathrm{~mm}$ & $50 \mathrm{~mm}$ & $75 \mathrm{~mm}$ & $75 \mathrm{~mm}$ \\
$2 w$ & $1.9 \mathrm{~mm}$ & $2.3 \mathrm{~mm}$ & $2.8 \mathrm{~mm}$ & $3.5 \mathrm{~mm}$ & $1.9 \mathrm{~mm}$ \\
$A$ & $a_{1}$ & $a_{2}$ & $a_{1}$ & $a_{2}$ & $a_{2}$ \\
$\eta_{11}$ & $89.6 \%$ & $94.6 \%$ & $94.0 \%$ & $93.6 \%$ & $91.1 \%$ \\
$w_{0} / a$ & 0.52 & 0.63 & 0.69 & 0.70 & 0.54 \\
$L_{p}(\mathrm{~dB})$ & 0.98 & 0.8 & 1.52 & 0.74 & 0.65 \\
$L_{t}(\mathrm{~dB})$ & 2.13 & 1.27 & 1.83 & 1.75 & 2.4 \\
\hline
\end{tabular}

that Eq. (8) provides a reliable estimation of $L_{t}$ for wavelengths $>4.6 \mu \mathrm{m}$, while at shorter wavelengths additional contributions to the losses should be taken into account. The overall losses underestimation can be ascribed to the low spatial quality of the laser beams. Indeed, in the theoretical model we assumed a pure Gaussian optical power distribution of the laser beams at the HCW input. For nonGaussian input beam profiles, the amount of the optical power coupled with the higher-order HCW modes increases, giving rise to higher total losses. ${ }^{24}$ The larger discrepancy observed at $3.5 \mu \mathrm{m}$ is due to a dielectric layer for this wavelength, which is thicker than optimal and this effect is visible in Fig. 3.

\section{Conclusions}

In this paper, we reported a detailed study of the influence of the coupling lens $f$, the laser wavelength, and the input core size on the optical performance of a tapered HCW having a bore diameter linearly varying from 200 to $260 \mu \mathrm{m}$ over a length of $50 \mathrm{~cm}$. Theoretical calculations allow predicting the best operating conditions to achieve single-mode output and low optical losses as a function of the input laser beam wavelength. These theoretical predictions have been verified by optically coupling the HCW with an ICL and four QCLs emitting within the 3.5 to $7.8 \mu \mathrm{m}$ spectral ranges. Singlemode Gaussian-like beam profiles at the HCW exit were obtained for all the employed light sources, even if using the $a_{2}$ as the input side. This confirms that tapered HCWs allow using a larger bore size for single-mode operation with respect to standard $\mathrm{HCW}$, thereby facilitating the fiber-laser alignment. The reported experimental results validate the theoretical calculations that can be used as a valid tool to predict the optimal coupling lens $f$ providing the single-mode output and lowest losses at different wavelengths. All these analyses can also be extended to the case of standard HCWs.

\section{References}

1. W. Yang et al., "Atomic spectroscopy on a chip," Nat. Photonics 1, 331335 (2007).

2. A. Ksendzov et al., "Modal filtering for midinfrared nulling interferometry using single mode silver halide fibers," Appl. Opt. 47, 5728 (2008). 
3. R. M. Verdaasdonk and C. F. P. van Swol, "Laser light delivery systems for medical applications," Phys. Med. Biol. 42, 869-894 (1997).

4. R. Bernini, S. Campopiano, and L. Zeni, "Silicon micromachined hollow optical waveguides for sensing applications," IEEE J. Sel. Top. Quantum Electron. 8, 106-110 (2002).

5. J. A. Harrington, "A review of IR transmitting, hollow waveguides," Fiber Integr. Opt. 19, 211-227 (2000).

6. P. Patimisco et al., "Low-loss hollow waveguide fibers for mid-infrared quantum cascade laser sensing applications," Sensors 13, 1329-1340 (2013).

7. J. A. Harrington, Infrared Fibers and Their Applications, SPIE Press, Bellingham, Washington (2004)

8. C. M. Bledt, J. A. Harrington, and J. M. Kriesel, "Loss and modal properties of Ag/AgI hollow glass waveguides," Appl. Opt. 51, 3114 (2012).

9. A. Sampaolo et al., "Single mode operation with mid-IR hollow fibers in the range $5.1-10.5 \mu \mathrm{m}$," Opt. Express 23, 195 (2015).

10. M. S. de Cumis et al., "Widely-tunable mid-infrared fiber-coupled quartz-enhanced photoacoustic sensor for environmental monitoring," Opt. Express 22, 28222 (2014).

11. V. Spagnolo et al., "Part-per-trillion level $\mathrm{SF}_{6}$ detection using a quartz enhanced photoacoustic spectroscopy-based sensor with single-mode fiber-coupled quantum cascade laser excitation," Opt. Lett. 37, 4461 (2012).

12. V. Spagnolo et al., "Mid-infrared fiber-coupled QCL-QEPAS sensor," Appl. Phys. B 112, 25-33 (2013).

13. P. Patimisco et al., "Low-loss coupling of quantum cascade lasers into hollow-core waveguides with single-mode output in the 3.7-7.6 $\mu \mathrm{m}$ spectral range," Sensors 16, 533 (2016).

14. P. Patimisco et al., "Hollow core waveguide as mid-infrared laser modal beam filter," J. Appl. Phys. 118, 113102 (2015).

15. J.M. Kriesel et al., "Hollow core fiber optics for mid-wave and longwave infrared spectroscopy," Proc. SPIE 8018, 80180V (2011).

16. A. Sampaolo et al., "Highly sensitive gas leak detector based on a quartz-enhanced photoacoustic SF6 sensor," Opt. Express 24, 15872 (2016).

17. R. Nubling and J. A. Harrington, "Launch conditions and mode coupling in hollow glass waveguides," Opt. Eng. 37, 2454 (1998).

18. C. M. Bledt, J. E. Melazer, and J. A. Harringtion, "Theoretical and experimental investigation of infrared properties of tapered silver/silver halide-coated hollow waveguides," Appl. Opt. 52, 3703 (2013).

19. E. A. J. Marcantili and R. A. Schmeltzer, "Hollow metallic and dielectric waveguides for long distance optical transmission and lasers," Bell Syst. Tech. J. 43, 1783-1809 (1964).

20. Y. Matsuura and M. Miyagi, "Hollow optical fibers for ultraviolet and vacuum ultraviolet light," IEEE J. Sel. Top Quantum Electron. 10, 1430-1434 (2004).

21. C. Dragone, "Attenuation and radiation characteristics of the HE11 mode," IEEE Trans. Microwave Theory Tech. 28, 704-710 (1980).

22. M. Miyagi and S. Kawakami, "Design theory of dielectric-coated circular metallic waveguides for infrared transmission," J. Lightwave Technol. 2, 116-126 (1984).

23. R. George and J. A. Harrington, "Infrared transmissive, hollow plastic waveguides with inner Ag-Agl coatings," Appl. Opt. 44, 6449 (2005)

24. Y. Matsuura et al., "Loss characteristics of circular hollow waveguides for incoherent infrared light," J. Opt. Soc. Am. A 6, 423 (1989).

Marilena Giglio received her MS degree (cum laude) in applied physics from the University of Bari, Bari, Italy, in 2014, where she is currently pursuing her PhD in physics. Since September 2016, she has been a research assistant in the Laser Science Group at Rice University, Houston, Texas. Her research interests include development of gas sensors based on quartz-enhanced photoacoustic spectroscopy and study of optical coupling of waveguides in the mid-IR spectral range.

Pietro Patimisco received his master's (cum laude) and PhD degrees from the University of Bari, Bari, Italy, in 2009 and 2013, respectively, both in physics. Since 2013 , he has been a postdoctoral research assistant at the University of Bari. He was a visiting scientist at the Laser Science Group, Rice University, Houston, Texas, USA, from 2013 to 2014. His research interests are focused on optical gas sensing in the midinfrared and terahertz spectral region.

Angelo Sampaolo received his master's degree and $\mathrm{PhD}$ from the University of Bari, Italy, in 2013 and 2017, respectively, both in physics. Since April 2017, he is research assistant with the University of Bari. His research interests include the study of the thermal properties of heterostructured devices via Raman spectroscopy, development of innovative techniques in trace gas sensing based on quartzenhanced photoacoustic spectroscopy and covering the full spectral range from near-IR to $\mathrm{THz}$.

Jason M. Kriesel is a lead scientist at OKSI, leading the development of hollow fiber optic research and the resulting commercial products. In addition, he is leading development of laser based mid-IR spectroscopy sensor technology at OKSI, including sniffers that utilize the hollow fiber as a small volume gas cell. He has been employed at OKSI since 2002, prior to this, he was an NRC postdoc in David Winelands group at NIST, Boulder.

Frank K. Tittel received his master's and doctorate degrees from the University of Oxford, Oxford, U.K., in 1955 and 1959, respectively, both in physics. Since 1967, he has been the Faculty of the Department of Electrical and Computer Engineering and Biomedical Engineering, Rice University, Houston, Texas, USA, where he currently an Endowed Chaired Professor. His research interests include laser spectroscopy and applications. He is a fellow of the OSA, APS, and SPIE.

Vincenzo Spagnolo received his $\mathrm{PhD}$ in physics from the University of Bari, Bari, Italy, in 1994. Since 2015, he has been an associate professor with the Department of Physics, Polytechnic of Bari, Bari, Italy. His research interests include quantum cascade lasers and optical gas sensing. He is the director of the joint-research Lab PolySense created by THORLABS GmbH and Technical University of Bari. He is a senior member of the SPIE. 